\title{
La catégorie de la réticence et un retour au récit dans l'écriture de Jean-Philippe Toussaint. Une introduction
}

\author{
The Category of Reticence and a Return to the Story in Jean-Philippe Toussaint's Writing. \\ An Introduction
}

\begin{abstract}
The article presents the function of reticence in Jean-Philippe Toussaint's writing in the context of the return to the story in francophone literature, initiated in the $80 \mathrm{~s}$ of the $20^{\text {th }} \mathrm{c}$. Jean-Philippe Toussaint is a contemporary Belgian writer, photographer and film-maker. He has written twelve novels and short stories, as well as authored films and installations. Belonging to the generation of minimalist writers, Toussaint sets his use of reticence in the context of the tendency to return to Genette's category of story (récit). Since the publication of his novel $\mathrm{La}$ Réticence (1991) reticence has become the key category of his écriture. In his books, it shapes the form, the narration and the plot, the construction of characters, temporality, and space. Thus, its function in Toussaint's writing enables us to observe idiosyncratic aspects of his "infinitesimalist" texts which play with the canonical, realistic model of the novel.
\end{abstract}

Keywords: francophone contemporary prose, Jean-Philippe Toussaint, reticence, return to the story

Mots-clés : littérature francophone de l'extrême-contemporain, Jean-Philippe Toussaint, réticence, retour au récit

Kategoria „powściagliwości” (réticence) i powrót do opowieści w pisarstwie Jeana-Philippe'a Toussainta. Wprowadzenie

Streszczenie: Artykuł stanowi próbę omówienia kategorii powściągliwości (fr. réticence) w prozie Jeana-Philippe'a Toussainta w kontekście powrotu do opowieści w literaturze frankofońskiej, zapoczątkowanego w latach 80 . XX wieku. Jean-Philippe Toussaint to współczesny belgijski pisarz, fotograf i filmowiec. Napisał m.in. dwanaście powieści i kilka zbiorów krótkich form, jest także autorem filmów i instalacji. Jako przedstawiciel pokolenia pisarzy minimalistycznych Toussaint osadza wykorzystanie powściągliwości w kontekście zjawiska powrotu do genette'owskiej kategorii opowiadania (fr. récit). Od czasu publikacji powieści $L a$ 
Réticence (1991) powściągliwość stała się kluczową kategorią Toussaintowskiego pisarstwa. W jego utworach kształtuje formę, narrację i fabułę, ale także konstrukcję postaci, czasowość i przestrzeń. W ten sposób status omawianej kategorii w twórczości Toussainta umożliwia analizę poszczególnych, a zarazem charakterystycznych dla tego twórcy aspektów textes infinitésimalistes, które podejmują grę z modelem balzakowskiej powieści realistycznej.

Słowa kluczowe: francuskojęzyczna proza współczesna, Jean-Philippe Toussaint, powściągliwość, powrót do opowieści

En 1991, avec la publication de son quatrième roman paru en version papier et intitulé La Réticence Jean-Philippe Toussaint, artiste contemporain belge francophone, auteur de douze romans, des textes intermédiatiques, des films, des installations et des recueils de formes brèves, a fait de la catégorie de la réticence l'élément central de son écriture et de son activité artistique ${ }^{1}$. Aux côtés des traits distinctifs du minimalisme toussaintien, la réticence constitue une des lignes principales des analyses de son œuvre 2 .

Dans le présent article notre objectif est d'analyser la catégorie de la réticence en tant que fondement d'un retour au récit visible dans les textes de Jean-Philippe Toussaint ${ }^{3}$. En premier lieu, nous voulons présenter les diverses dimensions de la réticence dans l'œuvre littéraire de Jean-Philippe Toussaint. Nous tenons à illustrer ce panorama des niveaux de la réticence à l'aide d'exemples puisés dans le roman que l'écrivain belge a publié en 1997, La Télévision. Ce livre, l'un des deux romans de Jean-Philippe Toussaint parus dans les années 1990, est un texte doté d'un statut particulier. En effet, sa publication suit directement celle de $L a$ Réticence (1991) et en même temps précède la parution, en 2000, du premier recueil des formes brèves de Toussaint intitulé Autoportrait (à l'étranger) ${ }^{4}$. Le plus long des romans écrits par l'auteur belge, La Télévision permet d'apercevoir les diverses dimensions de la réticence conçue en tant que mécanisme narratif et en tant qu'un des traits de la fiction. de surcroît, nous tenons à mettre en avant le caractère dynamique de l'écriture réticente de Jean-Philippe Toussaint qui est visible dans La Télévision et qui résulte en fait du dévoilement de nouvelles connotations de cette métacatégorie dans chaque nouveau récit. Notre second objectif est cependant d'esquisser le contexte du retour au récit dans la littérature contemporaine de la langue française. En troisième lieu, nous voulons définir le caractère du phénomène du retour au récit dans l'écriture de Toussaint, un retour dans lequel la réticence est une notion clés 5

${ }^{1}$ Le site internet de Jean-Philippe Toussaint, http://www.jptoussaint.com/france.html (accès : 11.12.2020); J.-Ph. Toussaint, La Réticence, Paris 1991, p. 7-159.

2 J.-L. Hyppolite, Jean-Philippe Toussaint : Reticent Narratives [dans :] idem, Fuzzy Fiction, Nebraska 2006, p. 24-61; R. Amar, Dispositifs minimalistes ou réticents? [dans :] Les Vérités de JeanPhilippe Toussaint, dir. S. Chaudier, Saint-Étienne 2016, p. 189-199.

${ }^{3}$ F. Shoots, «Passer en douce à la douane ». L'Écriture minimaliste de Minuit : Deville, Echenoz, Redonnet et Toussaint, Amsterdam-Atlanta, GA 1997, p. 101-139.

${ }^{4}$ Le site internet de Jean-Philippe Toussaint, http://www.jptoussaint.com/france.html (accès : 25.03.2021); J.-Ph. Toussaint, La Télévision, Paris 1997, p. 7-270.

${ }^{5} \mathrm{H}$. Godard, Le roman modes d'emploi, Paris 2006, p. 11-30. 
Étant donné que Jean-Philippe Toussaint est considéré par Jacques-Pierre Amette comme « le porte-drapeau » du minimalisme de la maison d'édition Minuit $^{6}$, il est à supposer que certains traits essentiels de la littérature minimaliste se laissent observer dans ses textes. Parmi ces caractéristiques fondamentales Warren Motte énumère notamment des récits concis et ordonnés, une tonalité froide et désaffectée, le caractère elliptique de l'écriture, des intrigues superflues et réduites ${ }^{7}$. Cette perspective qui met en avant les caractéristiques telles que le manque, l'ordre et la brièveté semble inscrire l'écriture de Toussaint dans la grille d'analyse proposée par John Barth qui, au sein du minimalisme littéraire, distingue le minimalisme formel, stylistique et celui du contenu narratif ${ }^{8}$. Pourtant, il est à ajouter, avec Ruth Amar, que seuls quelques-uns des critères cités par Motte, comme réduction de l'intrigue, clarté ou caractère ordonné du texte, sont adéquats aux récits de Jean-Philippe Toussaint ${ }^{9}$. En revanche, la concision et le caractère elliptique du langage, ainsi qu'un manque de profondeur au niveau de la construction de l'univers représenté, découlent de l'usage de la réticence. Cette dernière catégorie fait fonctionner le minimalisme littéraire toussaintien tout en $«$ en corrigeant les limites $»^{10}$.

Sans réfuter le caractère minimaliste de l'écriture de Toussaint, la réticence en fait pourtant "l'écriture infinitésimaliste ", une écriture fondée sur les oxymores $^{11}$. Jean-Philippe Toussaint, plus encore qu'un écrivain minimaliste, se définit en tant qu'auteur qui postule « de façon sous-jacente, (...) une littérature centrée sur l'insignifiant, sur le banal, le prosaïque $»^{12}$. Tandis qu'il construit l'univers représenté de ses récits sur de petits détails de la vie quotidienne, sur le " pas intéressant, pas édifiant ", en même temps ces " événements en marge » rencontrent toujours, au niveau de la forme, la perspective 4 maximaliste $»^{13}$. En effet, les textes toussaintiens tels que L'Appareil-photo (1988), La Réticence (1991), La Mélancolie de Zidane (2006) ou M.M.M.M. (tétralogie qu'il a écrit entre 2001 et 2013 pour la républier en un seul volume en 2017) s'ouvrent de plus en plus « à la dépense et à la surcharge », à « l'en-trop, l'excès »" La valorisation du maximal devient cependant possible grâce à l'esthétique de la réticence. Cette dernière introduit dans les textes de Jean-Philippe Toussaint des " échos de l'infiniment petit au sein de l'infiniment grand (une dynamique d'effet papillon) », « le microscopique [qui] côtoie l'infini », pour annoncer une suite de la fiction qui ne pourra jamais se réaliser pleinement, mais qui invite à interpréter

${ }^{6}$ F. Shoots, op. cit., p. 16-17.

7 W. Motte, Small Worlds. Minimalism in Contemporary French Literature, Nebraska 1999, p. 23.

${ }^{8}$ F. Shoots, op. cit., p. 52-56.

${ }_{9}$ R. Amar, op. cit., p. 190-191.

${ }^{10}$ Ibid., p. 191.

${ }^{11}$ L. Demoulin, Pour un roman infinitésimaliste [dans :] J.-Ph. Toussaint, L'Appareil-photo, Paris 1988/2007, p. $140-141$.

12 Ibid., p. 136.

${ }^{13}$ Ibid.; L. Ruffel, Le minimal et le maximal ou le renouvellement [dans :] Romanciers minimalistes 1979-2003, dir. M. Dambre, B. Blanckeman, Paris 2012, p. 43-54.

${ }^{14}$ Ibid., p. 43-45; Le Trop et le trop peu, une esthétique des extrêmes, dir. P. Ouellet, S. Pépin, Montréal 1999, p. 11, cité selon L. Ruffel, op. cit., p. 43. 
et combler un vide ${ }^{15}$. de cette façon, les contraires deviennent complémentaires, la patience et l'urgence que Toussaint perçoit en tant que sources de chaque écriture créent un récit « infinitésimaliste $»^{16}$. «Les deux infinis qu'on devrait toujours trouver dans les livres », le peu et le trop qui «n'ont pas l'habitude d'être traités » par les écrivains, dans les récits de Toussaint, englobent et en même temps dépassent le qualificatif " minimaliste » que l'écrivain belge trouve " quand même très réducteur $\rrbracket^{17}$.

Plus que sur la « réduction des conventions et [des] techniques modernistes » au « minimal element of (...) particular art », sur le « recours au minimum » et à la brevitas, les textes de Jean-Philippe Toussaint sont fondés sur la suggestion $^{18}$. L'impression d'un manque de contenu au niveau de l'histoire présentée et la réduction des procédés littéraires ne s'avèrent ici qu'apparentes. Dans les récits de l'auteur belge, la réduction minimaliste est un langage censé souligner des éléments apparemment manquants, si l'on compare ces textes au canon du roman traditionnel. L'écrivain belge commente ce choix dans les termes suivants : « ce qu'il y a de plus fort, dans un roman, c'est ce qui manque ${ }^{19}$. de cette manière, ce qui est mis en avant dans le récit de Jean-Philippe Toussaint, c'est le rôle des « lieux d'indétermination » dont Roman Ingarden avait constaté l'existence dans chaque fiction ${ }^{20}$.

Comme on le sait, dans la conception ingardenienne, chaque œuvre littéraire est une construction fondée sur quatre couches : la couche des formations phoniques du langage, la couche des unités de signification, la couche des « objets » figurés et la couche des aspects schématisés ${ }^{21}$. Toutefois, leurs spécificités et le caractère du lien qu'elles mantiennent les unes avec les autres découlent plutôt de l'existence d'une autre dimension encore, celle « de l'unité de sens $»^{22}$. Au niveau des objets, le philosophe distingue les objets réels et les objets figurés. Tandis que les premiers sont individuels et « univoquement déterminés », les seconds sont indéterminés, «purement intentionnels $»^{23}$. La « nature schématique » des objets figurés se caractérise par la présence dans une œuvre des traits « posi-

${ }^{15}$ Ch. Meurée, Le temps à l'épreuve du «désastre infinitésimal » [dans :] Les Vérités..., op. cit., p. 201, 208, 210 ; R. Amar, op. cit., p. 192.

${ }^{16}$ L. Demoulin, op. cit., p. 139-141; Ch. Meurée, op. cit., p. 210.

17 R. Amar, op. cit., p. 190-196 ; L. Demoulin, op. cit., p. 135-141.

${ }^{18}$ F. Shoots, op. cit., p. 51, 52; T. D'haen, Postmodernism in American Fiction and Art [dans :] Approaching Postmodernism, dir. D.W. Fokkema, H. Bertens, Amsterdam-Philadelphia 1986, p. 217 , cité selon F. Shoots, op. cit., p. 51.

${ }^{19}$ N. Kaprèlian, Rencontre avec Jean-Philippe Toussaint, https://www.lesinrocks.com/2009/09/25/ actualite/actualite/rencontre-avec-jean-philippe-toussaint/ (accès : 11.12.2020), cité selon J.-B. Gabriel, Fuir l'image avec désinvolture. Autour du court métrage Fuir de Jean-Philippe Toussaint, « Textyles : revue des lettres belges de la langue française » 2010, $\mathrm{n}^{\circ} 38$, https://journals.openedition.org/ textyles/289 (accès : 11.12.2020).

${ }^{20}$ W. Rapak, Entre langage et silence - discours allusif [dans :] L'allusion en poésie, dir. J. Lajarrige, Ch. Moncelet, Clermont-Ferrand 2002, p. 49-61.

${ }^{21}$ R. Ingarden, L'œuvre d'art littéraire, Lausanne 1983, p. 43, cité selon W. Rapak, op. cit., p. 49-61.

${ }^{22}$ Ibid.

${ }^{23}$ Ibid., p. 209-216. 
tivement attribués » et des « lieux d'indétermination $»^{24}$. Ces derniers passent souvent inaperçus dans de différents textes. Certain de ces lieux d'indétermination sont complétés dans le cours de la lecture. Toute œuvre demande à ce que ses lieux d'indétermination soient comblés par les lecteurs ${ }^{25}$. Pourtant, ce processus «ne peut jamais, quant au texte, (...) être men[é] à terme $»^{26}$.

Dans la pratique littéraire de Jean-Philippe Toussaint, ce sont ces indéterminations ingardeniennes qui jouent le rôle primordial. Au niveau de la fiction, la signification demande à être constamment complétée. Ce mécanisme résulte, comme c'est d'ailleurs le cas dans tous les textes littéraires, en plusieurs lectures possibles du livre et en caractère plus ou moins inachevé de l'œuvre ${ }^{27}$. Toutefois, Toussaint construit le tissu romanesque surtout à l'aide d'indéterminations, tout en mettant en avant ce qui, dans l'œuvre littéraire, reste d'ordinaire inaperçu ${ }^{28}$. Pour l'auteur belge, l'interprétation du texte consiste davantage en une herméneutique d'inachevé, du fragmentaire et du vide, qu'en une lecture des éléments déterminés, univoques ou facilement déchiffrables. Si un plein accomplissement de la signification s'avère impossible puisque le texte laisse toujours un espace pour l'indétermination ${ }^{29}$, pourtant, dans le récit toussaintien une suspension et une attente se produisent pour souligner l'importance d'une nouvelle tentative d'interprétation. Ainsi, la réticence transforme la réduction minimaliste en une quête, en une recherche constante ${ }^{30}$.

Jean-Philippe Toussaint souligne que le caractère polysémique du mot français « réticence » le fascine ${ }^{31}$. Pour lui, la réticence signifie :

ne pas avoir très envie (...), bref une sorte de mollesse dans le refus. Ce sens-là, il est évident, il me plaît (...), mais il y en a aussi un autre qui m'intéresse : le manque, la chose qui n'est pas dite, l'omission ${ }^{32}$.

Pour l'écrivain belge, la réticence est donc un procédé spécifique qui place les possibilités et les limites de l'expression littéraire au centre d'une écriture. La dimension « conceptuelle » de cette catégorie la transforme en noyau du programme créatif toussaintien ${ }^{33}$. Il est à supposer que c'est la réticence qui constitue le principe et la condition sine qua non de l'écriture de Jean-Philippe Toussaint ${ }^{34}$.

Ce principe s'appuie, au niveau de la narration, sur une stratégie narrative spécifique et, au niveau du contenu narratif, sur la création de l'univers représenté qui s'efface constamment et qui s'avère caractéristique pour les textes de Tous-

${ }^{24}$ Ibid., p. 213-214.

${ }^{25}$ Ibid.

${ }^{26}$ Ibid.

${ }^{27}$ Ibid.

${ }^{28}$ Ibid.

29 Ibid., p. 209-216.

${ }^{30}$ R. Amar, op. cit., p. 192-198.

${ }^{31}$ R.-M. Allemand, Jean-Philippe Toussaint : la forme et la mélancolie, « analyses » 2011, vol. 6, $\mathrm{n}^{\circ} 1$, p. 389

32 Ibid.

${ }^{33}$ Ibid.

${ }^{34}$ R. Amar, op. cit., p. 191-196. 
saint ${ }^{35}$. Ce procédé littéraire de la réticence semble se déployer en parallèle avec la conception toussaintienne de l'art et de la création littéraire en particulier. Pour l'auteur belge, comme il le signale par ailleurs dans plusieurs interviews, la réticence devient une métaphore de la littérature et de l'art qui se construisent sur l'implicite, entre les lignes ${ }^{36}$. Cette perspective adoptée par l'écrivain en question trouve ses fondements dans la volonté de « rendre hommage aux livres sans passer par l'écrit $\gg{ }^{37}$. Elle se traduit dans la pratique scripturale de Jean-Philippe Toussaint. Le processus de l'interruption et de l'omission des plusieurs éléments que ses textes mettent en place, le refus de dire, d'exprimer les choses ouvertement par moyens du langage, semble relever d'une pratique allusive, d'un travail de suggestion, qui s'avèrent des éléments cruciaux de l'écriture réticente ${ }^{38}$. Dans les récits de l'artiste belge, le sous-entendu et l'implicite sont complémentaires de la dimension rhétorique de la réticence qui se fonde sur l'usage de différentes figures du silence. Ce sont avant tout des figures par détraction : reticentia, praeteritio, abruptio, ou encore aposiopesis ${ }^{39}$. Fondée sur la réticence, l'écriture de Jean-Philippe Toussaint mène son propre « jeu avec le silence ${ }^{40}$.

Dans Le Petit Robert de la Langue française, il est à retrouver quatre définitions de la réticence. Ce mot dont l'étymologie se lie au verbe latin reticere rester tû et le substantif reticentia - silence obstiné, peut signifier « omission volontaire d'une chose qu'on devrait dire, chose omise » ou " dissimulation d'un fait $\gg{ }^{41}$. Pour notre propos, ce sont néanmoins les deux autres définitions qui prennent de l'importance, puisqu'elles éclairent le caractère et les causes des réticences toussaintiennes. En effet, la réticence est une figure de rhétorique «par laquelle on interrompt brusquement la phrase, en laissant entendre ce qui suit » et en même temps elle peut désigner une " attitude ou [un] témoignage de réserve, de doute, d'hésitation, dans les discours, le comportement $»^{42}$. Déjà ces définitions montrent la polysémie de la réticence, notion qui, dans l'usage qu'en fait Jean-Philippe Toussaint, dépasse sa dimension rhétorique.

Ruth Amar évoque trois définitions de la réticence dans le champ rhétorique. Ainsi, Antoine-Jeudy Dugour nous rappelle que la réticence a lieu quand « l'orateur rompt tout à coup le fil de son discours $\rangle^{43}$. D'après Quintilien, la réticence, avec l'aposiopesis ou encore l'interruptio, appartient au répertoire plus large des figures qui se distinguent par un vide difficile à remplir, crée par l'interruption

${ }^{35}$ Ibid., p. 191-196, 198.

${ }^{36}$ Ibid., p. 191-199.

${ }_{37}$ J.-Ph. Toussaint, La Main et le Regard. LIVRE/LOUVRE, Paris 2012, p. 18.

${ }^{38}$ W. Rapak, op. cit., p. 49-61 ; R. Amar, op. cit., p. 191.

${ }^{39}$ W. Rapak, op. cit., p. 49-61 ; R. Amar, op. cit., p. 191-194, 197-199.

40 W. Rapak, op. cit., p. 49-61.

${ }^{41}$ Le Petit Robert de la Langue française, 2011, informatisé, entrée " réticence », disque CD (accès : 11.12.2020).

${ }^{42}$ Ibid.

${ }_{43}$ A.-J. Dugour, Nouvelle Rhétorique française à l'usage des jeunes demoiselles, Bossange 1792, p. 261, http://gallica.bnf.fr/ark:/12148/bpt6k49071g (accès : 11.12.2020), cité selon R. Amar, op. cit., p. 191-192. 
subite de la parole ${ }^{44}$. Cependant, la spécificité de la réticence consiste à introduire dans l'énoncé une sorte d'incertitude, un doute que le récepteur est censé dissiper en devinant la suite entre les lignes ${ }^{45}$. Par ailleurs, Vladimir Jankélévitch complète cette définition antique en mettant en avant le fait que la réticence est une figure de silence semblable à la pause dans une composition musicale qui implique une « interruption expressive, le discours expirant, le passage de l'explicite au tacite $»^{46}$. C'est pour cette raison, comme le souligne Pierre Fontanier, que la réticence, par interruption du propos, peut « faire entendre (...) ce qu'on affecte de supprimer, et même souvent beaucoup au-delà $\gg{ }^{47}$. La réticence dans son aspect rhétorique, ainsi que dans sa dimension liée à l'attitude pleine d'émotions est reflétée dans la construction de l'univers représenté et dans le mode de la narration adoptés par Jean-Philippe Toussaint.

Le niveau diégétique des textes toussaintiens se construit sur le principe de la réticence, visible dans le caractère des personnages, des péripéties, de l'espace et de la temporalité. Le protagoniste qui, dans la plupart des livres, est un le narrateur autodiégétique ${ }^{48}$, s'avère le plus souvent un homme trentenaire anonyme. Son indétermination en tant que personnage se manifeste non seulement par le fait qu'il n'a ni nom de famille ni prénom, mais aussi à travers l'absence de repères approfondis quant à ses relations familiales, son éducation ou son travail professionnel. Même le personnage éponyme du roman Monsieur (1986), le seul à ne pas être le narrateur du récit et l'un de trois protagonistes toussaintiens désignés par une sorte de prénom ${ }^{49}$, quoique nommé, devient encore plus un everyman indéterminé. L'absence des traits caractéristiques de l'apparence, de l'identité ou de la profession du personnage s'entrelace avec son attitude spécifique à la réalité. Comme le note en effet Audrey Camus, le protagoniste toussaintien fait de la réticence sa « manière d'être au monde » ${ }^{50}$.

Les narrateurs de Jean-Philippe Toussaint se caractérisent majoritairement par un comportement distancié par rapport au réel. Ils se contentent de leur oisiveté. Ils préfèrent s'abriter dans de petits espaces, comme des salles de bain, des toilettes publiques ou des cabines téléphoniques ${ }^{51}$. Leur retrait s'avère pourtant une réaction à la réalité qui leur pèse. Leurs réactions qui relèvent d'une volonté d'éviter la confrontation avec le monde ou plus encore de la reporter dans le temps, sont souvent liées à des émotions négatives telles que la peur, l'angoisse, la timidité, la gêne ou l'irritation ${ }^{52}$. Toutefois, le « personnage impassible » de Toussaint es-

${ }^{44}$ Quintilien, Institutio oratoria, IX, III, p. 61, cité selon R. Amar, op. cit., p. 192 ; D. Korwin-Piotrowska, Białe znaki. Milczenie w strukturze i znaczeniu utworów narracyjnych, Kraków 2015, p. 104.

${ }^{45}$ Ibid.

${ }^{46}$ V. Jankélévitch, L'Ironie, Paris 1964 [1950], p. 89, cité selon A. Camus, L'art de la conversation impossible [dans :] Les Vérités..., op. cit., p. 84.

${ }^{47}$ P. Fontanier, Les figures du discours, Paris 1977, p. 135, cité selon R. Amar, op. cit., p. 192.

${ }^{48}$ A. Richir, Écriture du fantasme chez Jean-Philippe Toussaint et Tanguy Viel. Diffraction littéraire de l'identité, Louvain 2015, p. 90-92.

${ }^{49}$ Ibid.

${ }^{50}$ A. Camus, op. cit., p. 84-87.

${ }^{51}$ J. Zhao, L'éloge de l'oisiveté [dans :] Les Vérités..., op. cit., p. 93-99.

52 A. Camus, op. cit., p. 84-90. 
saie le plus souvent de ne pas montrer ses émotions ${ }^{53}$. Induit dans une situation extrême, il transforme pourtant son silence réservé en impertinence, arrogance, rage et agressivité ouvertes envers autrui, des sentiments qui peuvent se manifester verbalement et/ou physiquement. Les protagonistes des récits toussaintiens veulent imposer cette attitude réticente aux autres personnages ${ }^{54}$.

Le refus constitue la clé de cette stratégie éthique. Tout en refusant de quitter sa salle de bain ou son appartement, de partir en voyage ou de manger, le personnage construit par Toussaint se plonge dans son activité lente et oisive qui devient le cœur des actions potentielles qu'il projette ${ }^{55}$. Ce comportement consiste avant tout à explorer l'univers de ses propres pensées. Le personnage se plonge dans le monde intérieur pour contempler un autre versant de sa vie, marqué non pas par l'inertie, mais par le mouvement et l'activité de l'imagination. C'est la pensée qui permet au protagoniste de devenir une partie intégrante de l'écoulement du temps qui le menace. Seulement à travers la contemplation calme de l'univers intérieur et extérieur ainsi que grâce à l'appréciation des événements quotidiens prévisibles il est possible d'arrêter ce flux dans un instant précis pour mieux en profiter ${ }^{56}$. Cette tension entre l'activité et l'oisiveté, entre le mouvement et l'immobilité est aussi reflétée dans la construction de l'espace et de la temporalité. Les personnages de Toussaint évoluent donc dans des espaces quotidiens, très souvent $\operatorname{clos}^{57}$. Ce sont des espaces indéterminés et périphériques, abîmés par le temps ou abandonnés, comme des banlieues (dans L'Appareil-photo), une décharge publique (La Réticence), un aéroport post-nazi (La Télévision). La temporalité, tout comme l'espace, reflète l'indétermination qui accompagne le protagoniste toussaintien. Les repères temporels s'avèrent brouillés. La perception du temps par les narrateurs oscille entre la joie des instants quotidiens et la durée imprécise qui découle de l'état d'âme changeant du protagoniste ${ }^{58}$. Le roman paru en 1997 illustre bien le principe de la réticence au niveau narratif et fictionnel dont Jean-Philippe Toussaint fait l'usage et en même temps montre l'évolution de cette catégorie.

La Télévision présente l'histoire d'un chercheur académique qui s'approche lentement de son quarantième anniversaire. Son prénom et son nom de famille ne sont pas mentionnés mais le protagoniste se décide à partir en Allemagne pour pouvoir profiter d'une bourse universitaire et rédiger une étude sur les relations entre les artistes et le monde de la politique. Arrivé à Berlin, au lieu de se concentrer sur la rédaction de son ouvrage, le protagoniste commence néanmoins à chercher le divertissement. Il arrête solennellement de regarder la télévision, mais surtout il passe son temps à discuter avec ses amis dans un café, à nager dans un lac ou dans une piscine, à visiter des musées. Parallèlement, il essaie de trou-

${ }^{53}$ F. Shoots, op. cit., p. 45-49.

54 A. Camus, op. cit., p. 84-90.

55 Ibid., p. 85.

56 R. Amar, op. cit., p. 196.

57 J. Zhao, op. cit., p. 93-95.

58 A. Richir, Capter l'image : l'expérience du temps dans «L'Appareil-photo » et " Faire l'amour » [dans :] Les Vérités..., op. cit., p. 181-187. 
ver le plus de temps possible pour le consacrer à son fils et à Delon, sa femme enceinte. Il est à noter que, comparé avec le roman précédent, La Réticence, qui puisait dans l'esthétique du roman policier du type noir et dans lequel le narrateur s'avèrait paralysé par ses réticences constantes et induit dans la paranoïa, de son côté, La Télévision présente une vision plus nuancée de l'univers marqué par le mécanisme de la réticence ${ }^{59}$.

Le narrateur autodiégétique de La Télévision est un homme anonyme mais en même temps enraciné dans un milieu universitaire concret et profondément lié à sa famille. S'il décide de reporter la rédaction de son essai dans le temps, c'est en raison de sa conviction selon laquelle :

(...) finalement, dans la perspective même d'écrire, ne pas écrire est au moins aussi important qu'écrire. (...) j'essayais de me consoler (...) en pensant que, paradoxalement, c'est plutôt si je m'étais mis à écrire tout de suite, (...) qu'on aurait pu me soupçonner de vouloir me dérober à l'effort pour me la couler douce à Berlin cet été, et qu'il y avait plutôt lieu de se réjouir, dans le fond, que, depuis bientôt trois semaines, (...) je m'étais ainsi contenté de me disposer en permanence à écrire, sans jamais céder à la paresse de m'y mettre ${ }^{60}$.

Ainsi, le protagoniste réfléchit à son activité scripturale tout en présentant la double nature de son attitude réticente envers le monde. En effet, dans l'univers représenté de La Télévision, la réticence ne se borne plus à l'action d'échapper au réel et à autrui. Si le narrateur cherche toujours de nouveaux prétextes pour se consacrer aux activités qui lui procurent du plaisir, il le fait pour mieux profiter de la vie, du monde qui l'entoure et de ses relations avec d'autres personnages. Il repousse dans le temps le moment du retour au travail mais il ne veut plus se cacher des autres. Quand sa femme part en vacances en Italie, il attend les conversations téléphoniques avec elle, même si celles-ci consistent majoritairement à écouter le silence de l'autre et à essayer d'évoquer son visage :

Delon (comme c'était bon d'entendre la voix réconfortante de Delon tout près de moi dans l'écouteur), sans même me demander de nouvelles de mon travail, me dit immédiatement qu'elle se sentait merveilleusement bien aujourd'hui, et que, pour la première fois, ce matin, elle avait senti la petite bouger (...). (...) Elle se tut, et je les imaginais nager toutes les deux dans une mer immobile, très bleue et transparente $(\ldots)^{61}$.

Dans la vie du narrateur du roman en question, la réticence constitue donc une opportunité de partager son temps libre avec l'autre mais avant tout de se sentir libre, ainsi que de se connaître mieux lui-même et son attitude envers la création artistique. Toutes les activités qu'il entreprend (rencontres, promenades, nage, sieste dans un parc) s'avèrent pour le personnage des occasions de réfléchir à l'importance de « cette lente et progressive ouverture de l'esprit et cette totale disponibilité des sens » dans le processus créatif ${ }^{62}$. Ainsi, dans La Télévision, la volonté de fuir le monde et d'imposer le silence aux autres est-elle contre-

${ }^{59}$ S. Glasco, Parody and Palimpsest. Intertextuality, Language and the Ludic in the Novels of Jean-Philippe Toussaint, New York 2015, p. 95-99.

${ }^{60}$ J.-Ph. Toussaint, La Télévision, op. cit., p. 90, 115.

${ }^{61}$ Ibid., p. 102-103.

${ }^{62}$ Ibid., p. 89. 
balancée par le désir profond d'un contact avec autrui et par un regard ironique envers soi-même et envers la réalité :

$\mathrm{D}$ [éjà] un début de phrase m'était venu en chemin en revenant du parc. Je me répétai la phrase mentalement (...). Quand Musset, répétai-je à voix basse. Non, rien à dire, c'était un bon début. Je le dis un peu plus fort. (...) Quand Musset ! répétais-je au balcon. Quand Musset ! Silence ! entendis-je soudain, silence, s'il vous plaît ! (...) C'était le propriétaire (...). Je soulevai mon chapeau poliment pour le saluer (...). (...) le gardien s'avança soudain vers moi à grands pas en hurlant de m'éloigner du tableau. Oui, oui, lui dis-je en m'éloignant du tableau (essayez de ne pas vociférer, ajoutai-je, n'oubliez pas que vous parlez allemand, tout de même $)^{63}$.

Il est aussi à souligner que le narrateur de La Télévision puise l'exemple de son attitude réticente dans la contemplation de l'art qui, avec la nage, paraît constituer son activité préférée. Le portrait de l'empereur Charles V peint par Titien lui sert de modèle de la réticence conçue en tant qu'un mélange d'émotions contradictoires : calme, sérénité et inquiétude :

J'étais immobile dans mon fauteuil, pensif (...). Presque tout, à ce moment-là, dans mon attitude, évoquait Charles Quint, je trouvais, le Charles Quint fatigué de la Pinacothèque de Munich, le visage pâle et pathétique, un gant à la main (...), le corps digne et las (...). De quoi avions-nous peur si sereinement ? ${ }^{64}$

La façon de construire le personnage conditionne ensuite l'intrigue et l'espacetemps présentés dans La Télévision. Le récit présente la vie quotidienne du narrateur qui part à l'étranger. L'intrigue se compose donc d'activités simples et banales que le protagoniste réalise de façon systématique et qui lui plaisent bien qu'elles consistent principalement à « ne rien faire (...), ne faire que l'essentiel, penser, lire, écouter de la musique, faire l'amour, [se] promener, aller à la piscine, cueillir des champignons $»^{65}$. Seules les visites dans les galéries d'art, le temps consacré à jouer avec son fils ou aux conversations avec sa femme, ainsi que le vol au-dessus de Berlin avec un ami rompent ce rythme répétitif du quotidien du narrateur scandé par des promenades, des déjeuners, des tâches ménagères et la nage.

Le roman commence par la décision du protagoniste d'arrêter de regarder la télévision. L'image du poste allumé ou déconnecté devient l'image centrale du livre qui, au moyen de la répétition, intensifie encore le rythme monotone de l'intrigue. L'impression d'une durée se crée en dépit de celle d'une progression temporelle. de cette façon, la lente progression de son existence quotidienne fait évoluer le narrateur comme en dehors de la temporalité. Néanmoins, la durée introduite par la réticence n'exclut pas complètement la progression de l'intrigue. Même si cette progression n'est possible que de façon partielle, elle se fonde, paradoxalement, sur la construction du personnage. En effet, l'attitude réticente du protagoniste constitue un type d'attente, de préparations à la suite des événements et aux activités qu'il veut mettre en œuvre. Ainsi, la réticence

\footnotetext{
${ }^{63}$ Ibid., p. 101-102, 231-232.

${ }^{64}$ Ibid., p. 51-52.

65 Ibid., p. 11.
} 
ouvre pour le narrateur de La Télévision la possibilité de « savourer » un « instant d'éternité » unique ${ }^{66}$.

Le mode de la représentation du cadre spatial qui se compose notamment des rues berlinoises vides, d'un aéroport désaffecté et d'une banlieue laide, sale et pleine des «blocs de béton identiques 》 contraste avec la routine quotidienne du protagoniste mais d'un autre côté, cette représentation découle aussi de manière de voir le réel qui est propre au personnage ${ }^{67}$. La vue de la ville à vol d'oiseau lors du passage en avion au-dessus de Berlin se distingue à son tour de la représentation anonyme, indéterminée de l'espace représenté tout en mettant en avant le caractère plurivoque de la réticence qui, dans La Télévision, se trouve dotée d'une valeur à la fois complémentaire et plus positive par rapport au roman précédent.

Cette construction réticente de l'univers représenté reflète la stratégie formelle et narrative adoptée dans le récit. Jean-Philippe Toussaint inscrit le silence dans ses textes littéraires par le moyen des blancs typographiques qui transparaissent entre des blocs de texte et qui se traduisent dans les « arrêts, fragmentations, ruptures et glissements " narratifs ${ }^{68}$. Dans le récit toussaintien la tension entre le dit et le non-dit est visible. En découlent, d'un côté, des omissions de sujets grammaticaux, un usage fréquent des points d'interrogation, des suspensions de la narration et la déstabilisation de la chronologie mais aussi, d'un autre côté, des allusions, des parenthèses et un ressassement qui produit un effet de la rapidité de l'écriture ${ }^{69}$. Dans La Télévision, l'écriture par paragraphes côtoie l'usage fréquent de répétitions et d'énumérations. La fragmentation du texte et la chronologie perturbée se trouvent encore renforcées par le recours, au niveau de la fiction, à des images concrètes répétées. Cette discontinuité tend pourtant à créer, de façon paradoxale, une impression de cohérence de l'histoire présentée. Le caractère apparemment lacunaire du texte dévoile le rôle du principe de la réticence qui met l'accent sur un autre récit émergent. Les absences apparentes dans le tissu narratif créent un récit parallèle et soulignent l'importance des sens sous-entendus que le lecteur peut librement faire surgir ${ }^{70}$. Ce récit virtuel alternatif, grâce à cette stratégie " réticente », visible à différents niveaux du texte, est à la fois un échec constant et un «méta-texte » qui ouvre «de nouveaux horizons pour le roman $»^{71}$.

Même si, en un certain sens, comme le remarque Ruth Amar, " le roman a perdu sa capacité de signifier », l'insistance sur les possibilités signifiantes de la réticence rend possible une recherche « de nouvelles formes d'écriture » par l'artiste belge ${ }^{72}$. Ainsi conçu, le principe de l'écriture réticente se lie à la métaphore toussaintienne de l'art et de la littérature. Si Robert Smithson, par ses sculptures

\footnotetext{
66 Ibid., p. 270.

67 Ibid., p. 195.

68 R. Amar, op. cit., p. 192.

69 Ibid., p. 191-196.

70 Ibid., p. 192-194.

71 Ibid., p. 196.

72 Ibid., p. 195, 196.
} 
minimalistes, développe la dialectique du site et du non-site qui met en valeur le matériau utilisé, Jean-Philippe Toussaint, par le moyen de la réticence, fonde sa métaphore de la création artistique sur les oxymores ${ }^{73}$. Entre le dit et le non-dit, entre les lignes, l'écrivain belge fait surgir un reflet du récit qui, de prime abord, paraissait inaccessible. de cette manière, l'écriture toussaintienne met en avant l'exploration du langage littéraire même ${ }^{74}$.

L'écriture de Jean-Philippe Toussaint s'inscrit cependant dans une tendance plus large dans la littérature d'expression française qui, selon Bruno Blanckeman, remonte jusqu'aux années 1980 , celle du $《$ retour du récit $\rrbracket^{75}$. Pour comprendre l'essentiel de ce retour que mettent en œuvre les textes des écrivains dits « maximalistes », ainsi que ceux des auteurs qualifiés de « minimalistes $»^{76}$, il faut évoquer les fondements de la version dite canonique du récit, le « récit mimétique $»^{77}$. À cet égard, Gérard Genette souligne l'ambiguïté de la catégorie du récit. Ce terme admet en effet trois acceptions ${ }^{78}$.

La plus fréquente de ces dimensions est le " récit proprement dit », récit en tant que « énoncé narratif, le discours oral ou écrit qui assume la relation d'un événement ou d'une série d'événements, (...) texte narratif $»^{79}$. Dans son deuxième sens, le récit signifie "l'histoire, la diégèse, [le] contenu narratif », donc " la succession d'événements, réels ou fictifs, qui font l'objet de ce discours, et leurs diverses relations, (...) ensemble d'actions et de situations considérées en elles-mêmes $»^{80}$. Le troisième et le plus ancien des sens met à son tour en valeur le récit en tant qu' « événement (...) qui consiste en ce que quelqu'un raconte quelque chose : l'acte de narrer pris en lui-même (...), l'acte narratif producteur $\|^{81}$. Comme l'écrit Fieke Shoots, le phénomène du retour au récit, la « renarrativisation » constatée par Aron Kibédi Varga, touche ce deuxième sens genettien et consiste en une " redécouverte actuelle de l'histoire, du roman et de l'imaginaire $»^{82}$. Il est ici à noter que l'histoire constitue l'un des points centraux du roman mimétique.

Selon une constatation d'Henri Godard, le modèle du roman dit canonique repose sur l'illusion romanesque qui consiste à faire passer les événements fictifs, racontés par le narrateur impersonnel, pour entièrement vrais, à « donn[er] une présence dans notre imagination à un monde qui imite notre monde sans

73 Ibid., p. 193, 194.

${ }^{74}$ Ibid., p. 192-194.

${ }^{75}$ B. Blanckeman, La littérature française au début du XXIe siècle : tendances en cours [dans :] M. Touret, Histoire de la littérature française, Rennes 2008, p. 443, cité selon M. Motkowicz, "La Salle de bain » de Jean-Philippe Toussaint en tant que roman minimaliste, mémoire de maîtrise non-publié, Cracovie 2011, p. 8.

${ }^{76}$ L. Ruffel, Le temps des spectres [dans :] Le roman français aujourd'hui. Transformations, perceptions, mythologies, dir. B. Blanckeman, J.-Ch. Millois, Paris 2004, p. 111-112.

${ }^{77}$ H. Godard, op. cit., p. 11.

${ }_{78}^{78}$ G. Genette, Figures III, Paris 1972, p. 71.

79 Ibid., p. 71, 72.

${ }^{80}$ Ibid.

${ }^{81}$ Ibid.

${ }^{82}$ F. Shoots, op. cit., p. 101; A. Kibédi Varga, Le récit postmoderne, « Littérature » 1990, n 77 , p. 153, cité selon F. Shoots, op. cit., p. 101. 
se confondre avec lui, (...) [en] nous [faire] vivre le déroulement de l'histoire et la vie des personnages $\gg{ }^{83}$. Le chercheur cité souligne le rôle primordial de l'histoire, du narrateur et du personnage dans le canon romanesque. D'après son opinion, tous les changements, inaugurés déjà par Gustave Flaubert et mis en œuvre en $\mathrm{XIX}^{\mathrm{e}}$ et $\mathrm{XX}^{\mathrm{e}}$ siècle en vue d'élaborer une " nouvelle poétique romanesque », étaient visés à remettre en question cette illusion référentielle ${ }^{84}$. C'est de «cet effet de fiction »spécifique que le roman dit canonique a fait sa «condition sine qua non ${ }^{85}$.

Il est à supposer que les écritures minimalistes promues dès la charnière des années 1970 et 1980 au sein des Éditions de Minuit, dont celle de Jean-Philippe Toussaint, ont avant tout pour but de remettre en question les notions clés de ce modèle mimétique, telles que l'histoire et le personnage ${ }^{86}$. Ce sont notamment ces deux catégories qu'Alain Robbe-Grillet classe parmi « quelques notions périmées » de la littérature ${ }^{87}$. Ce représentant du Nouveau Roman, tout en théorisant après coup les enjeux principaux de ledit courant, considère que « raconter est devenu proprement impossible ${ }^{88}$. En effet, l'acception balzacienne de l'histoire et du personnage romanesque semble s'épuiser avec l'arrivée du nouveau rapport entre l'homme et le monde, celui où l'être humain ne domine plus la réalité mais se retrouve au contraire soumis aux circonstances extérieures ${ }^{89}$.

Pour Alain Robbe-Grillet, être un « vrai romancier» implique surtout l'aptitude à raconter des histoires «palpitantes, émouvantes, dramatiques » et à créer des personnages auxquels le lecteur peut s'identifier ${ }^{90}$. à travers le roman mimétique un ordre concret du monde s'impose. La narration est menée à la troisième personne et au passé simple. La ligne d'intrigues tend vers une fin, la trame est régulière et les péripéties se développent dans des suites chronologiques. L'illusion référentielle s'ancre donc sur une vision stable, fiable et prévisible de la réalité. La continuité et la cohérence y règnent. L'univers représenté, dans ce modèle romanesque, est facilement déchiffrable ${ }^{91}$. Cette caractéristique de la fiction découle de la construction spécifique du personnage. Ce dernier est en effet un individu doté d'un caractère fort. Le lecteur peut s'identifier à lui grâce à son humanité, mais c'est aussi un personnage dont les traits servent de modèle pour un type humain plus général. Assez individuel et assez universel pour convaincre les lecteurs, ce protagoniste mène sa conquête du monde, scandée par ses ascensions et ses défaites. C'est une conception de l'univers dominé par l'homme enraciné dans son milieu. Quand cette vision « divine » change, l'histoire et le personnage deviennent des catégories périmées ${ }^{92}$. Alain Robbe-Grillet avance cependant

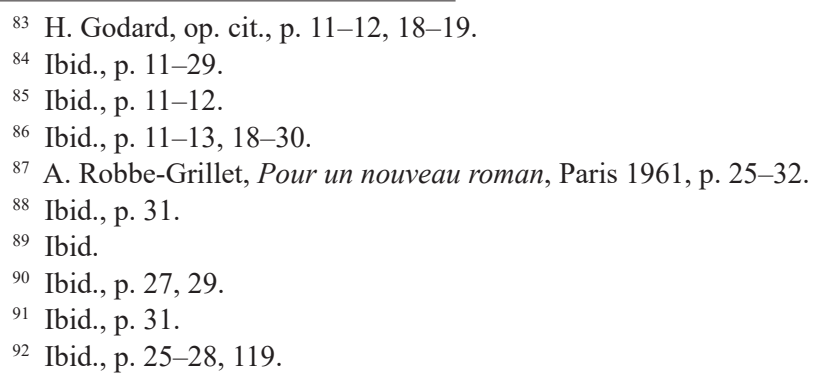


que, le modèle canonique devenu " idée toute faite, [et] donc idée morte ", l'histoire et les personnages ne doivent pas être soudain chassés de la littérature $^{93}$. L'évolution du monde qui nous entoure doit plutôt inviter les formes littéraires à changer elles aussi ${ }^{94}$. En effet, " le roman comme forme » est conçu par Robbe-Grillet en tant qu'une invention et une recherche ${ }^{95}$. C'est dans ce contexte que s'inscrit le retour au récit observable dans l'écriture de Jean-Philippe Toussaint.

Tout en parlant des traits caractéristiques du minimalisme des Éditions de Minuit, Aline Mura-Brunel l'inscrit dans le "schéma du retour », notion dont elle attribue l'origine à Jean-Luc Nancy ${ }^{96}$. L'auteure citée discute aussi le rôle des artistes dans cette tendance du retour ${ }^{97}$. Bruno Blanckeman, lui, inscrit « le retour au récit, au réel et au sujet » dans le cadre plus général du « retour du récit $»^{98}$. Nous sommes néanmoins d'accord avec Mura-Brunel pour dire que le récit ne s'impose de nouveau au sein de la littérature francophone dans sa variante minimaliste que grâce à l'activité consciente des écrivains concrets ${ }^{99}$. Pour cette raison, dans le présent article nous adoptons la notion du retour au récit. Bien que Dominique Viart, à raison, ne voie pas dans le récit minimaliste des tentatives du retour à une linéarité et à une chronologie de type réaliste, il est à noter que, dans ce jeu contemporain avec le modèle romanesque dit canonique, « la joie de la narration retrouvée » apparaît, paradoxalement, pour mettre en valeur la cohérence des histoires présentées ${ }^{100}$.

Comme le note Frank Wagner, dans les écritures minimalistes, dont celle de Jean-Philippe Toussaint, le retour au récit est possible notamment grâce à deux stratégies auctorielles : le recours à l'intertextualité et la mise en valeur du narrataire ${ }^{101}$. Il est surtout à observer que, dans les récits de Toussaint, les deux procédés évoqués sont subordonnés à la réticence. En effet, cette catégorie, d'un côté, introduit dans un livre la dimension métatextuelle et, d'un autre côté, pour citer la constatation de Ruth Amar, "l'ouvre à l'activité signifiante du récepteur $»^{102}$. Les narrateurs ne rompent pas l'illusion romanesque de façon directe et pourtant la réticence devient une marque du recours constant que fait JeanPhilippe Toussaint à ses livres précédents. Les histoires présentées, composées d'instantanés liés les uns aux autres de la façon arbitraire, puisent profondément

93 Ibid., p. 25, 32.

${ }^{94}$ Ibid., p. 114-120.

${ }^{95}$ Ibid., p. 114-115; H. Godard, op. cit., p. 15.

${ }^{96}$ A. Mura-Brunel, "Retour en grâce » de la fiction : "Un soir au club » de Christian Gailly et « Faire l'amour » de Jean-Philippe Toussaint [dans :] Romanciers minimalistes..., op. cit., p. 295-298, 301-302.

${ }^{97}$ Ibid., p. 302.

${ }_{98}$ B. Blanckeman, op. cit., p. 443, cité selon M. Motkowicz, op. cit., p. 8.

${ }_{99}$ A. Mura-Brunel, op. cit., p. 302.

100 D. Viart, B. Vercier, La littérature française au présent, Paris 2005, p. 17, cité selon M. Motkowicz, op. cit., p. 10 ; O. Bessard-Banquy, Le roman ludique, Villeneuve d'Ascq 2003, p. 21, cité selon A. Mura-Brunel, op. cit., p. 295.

${ }^{101}$ F. Wagner, Retours, tours et détours du récit. Aspects de la transmission narrative dans quelques romans français contemporains, « Poétique » 2011, n 165, p. 6-11, 14-17.

102 R. Amar, op. cit., p. 192. 
dans la vie quotidienne mais aussi dans l'esthétique du roman policier, du roman sentimental ou encore du roman d'aventures ${ }^{103}$. L'histoire se construit à chaque fois sous les yeux du lecteur pour mettre en avant sa valeur autoréférentielle. Des repères métatextuels accompagnent donc des " fausses anecdotes » pour faire du texte littéraire le sujet essentiel de la fiction ${ }^{104}$.

Ainsi, Toussaint thématise le travail de l'écriture et sa recherche de la réponse à la question de savoir qu'est-ce que, en réalité, la littérature contemporaine et comment le fait de raconter des histoires est-il possible. Dans cette écriture, le retour au récit s'opère, paradoxalement, à travers les tentatives vaines de définir celui-ci. à chaque fois, la catégorie de la réticence dévoile le caractère insaisissable du retour au récit qui constitue lui-même une réticence à l'égard de la chronologie, de la linéarité et de l'esthétique de l'avant-garde ${ }^{105}$. de cette façon, le retour au récit dans l'écriture de Jean-Philippe Toussaint ne peut se réaliser que grâce au déploiement du romanesque qui permet d' « interroger, revisiter et défaire le roman $\gg{ }^{106}$.

Pour récapituler, il est à souligner que, représentant du « Nouveau Nouveau Roman », Jean-Philippe Toussaint se réfère aux inventions romanesques des nouveaux romanciers tout en jouant avec le modèle dit balzacien du roman ${ }^{107}$. $\mathrm{Au}$ lieu d'une rupture, il choisit un jeu résultant en un retour à l'histoire qui se recherche elle-même. Toussaint, par l'usage des réticences, inscrit le silence dans ses récits ${ }^{108}$. Les absences apparentes se lient ici à une herméneutique de la fin proposée par Lionel Ruffel. Ainsi, l'auteur belge crée sa propre «spectralité » littéraire ${ }^{109}$. de cette façon, ses récits prennent appui sur une recherche romanesque tout en renonçant à la linéarité et à l'illusion réaliste. En raison de cette « déstabilisation douce », le modèle dit canonique n'est ici ni imité, ni tout à fait rejeté ${ }^{110}$.

Dans l'écriture de Jean-Philippe Toussaint, la tendance du retour au récit se réalise à travers le mécanisme de la réticence qui comporte une stratégie narrative et un mode de représentation particuliers ${ }^{111}$. L'auteur est conscient de l'héritage littéraire qui forme son écriture et avant tout des inventions du Nouveau Roman $^{112}$. Dans ses textes, sans le définir d'une manière univoque, Toussaint remet pourtant en question le problème du retour au récit. Il renonce à la rup-

${ }_{103}$ S. Glasco, op. cit., p. 95-133, 152-161, 169-189.

${ }_{104}$ M. Motkowicz, op. cit., p. 95.

${ }^{105}$ Ibid., p. 95-99.

106 S. Loignon, Romanesque : le retour de flamme, ou comment faire l'amour avec J.-P. Toussaint?

[dans :] Christian Oster et « Cie ». Retour du romanesque, dir. A. Mura-Brunel, Amsterdam-New York 2006, p. 27.

${ }^{107}$ F. Shoots, op. cit., p. 185-194.

${ }^{108}$ Ibid. ; R. Amar, op. cit., p. 191-196.

${ }^{109}$ L. Ruffel, Le temps ..., op. cit., p. 107-115.

110 Y. Leclerc, Autour de Minuit, «Dalhousie French Studies» 1989, Fall/Winter, p. 68, cité selon F. Shoots, op. cit., p. 193.

${ }^{111}$ Le site internet de Jean-Philippe Toussaint, http://www.jptoussaint.com/france.html (accès :

11.12.2020) ; B. Blanckeman, op. cit., p. 443, cité selon M. Motkowicz, op. cit., p. 8.

112 M. Motkowicz, op. cit., p. 8-11 ; F. Shoots, op. cit., p. 193-194. 
ture définitive avec le modèle dit canonique du roman, sans pour autant s'efforcer de l'imiter. Toussaint, à travers les absences apparentes, nous montre un autre récit potentiel qui émerge de ses textes. Il comprend donc le processus créatif en tant que changement dans la continuité ${ }^{113}$ :

Il est très important pour moi d'insister sur cette idée de continuité dans mon travail, même si je suis toujours attentif au renouvellement, même si je m'efforce de ne jamais refaire deux fois le même livre, même si écrire, pour moi, est toujours une recherche ${ }^{114}$.

Ainsi, pour Jean-Philippe Toussaint, le retour au récit s'avère un processus constant qui consiste en recherche de sa propre voix littéraire. Ce type de quête se trouve mis en avant dans les romans et de brefs textes successifs, tout en prévalant sur son propre résultat, sur le livre concret et achevé. La pratique scripturale réticente de Toussaint se réalise à travers des textes particuliers qui se substituent aux programmes et manifestes théoriques. Pour l'artiste belge, le retour au récit constitue donc un mode de dialogue avec la tradition romanesque et une stratégie permettant de mettre en valeur de différents « blancs » et « interstices » du texte littéraire ${ }^{115}$.

\section{Bibliographie (References)}

Allemand R.-M., Jean-Philippe Toussaint : la forme et la mélancolie, " analyses » 2011, vol. 6, $\mathrm{n}^{\mathrm{o}} 1$, p. 384-403.

Amar R., Dispositifs minimalistes ou réticents? [dans :] Les Vérités de Jean-Philippe Toussaint, dir. S. Chaudier, Saint-Étienne 2016, p. 189-199.

Bessard-Banquy O., Le roman ludique, Villeneuve d'Ascq 2003.

Blanckeman B., La littérature française au début du XXI siècle : profils culturels et La littérature française au début du XXIe siècle : tendances en cours [dans :] Histoire de la littérature française, dir. M. Touret, Rennes 2008, p. 429-490.

Camus A., L'art de la conversation impossible [dans :] Les Vérités de Jean-Philippe Toussaint, dir. S. Chaudier, Saint-Étienne 2016, p. 83-92.

Demoulin L., Pour un roman infinitésimaliste [dans :] J.-Ph. Toussaint, L'Appareilphoto, Paris 1988/2007, p. 129-141.

D'haen T., Postmodernism in American Fiction and Art [dans :] Approaching Postmodernism, dir. D.W. Fokkema, H. Bertens, Amsterdam-Philadelphia 1986, p. 211-231.

Dugour A.-J., Nouvelle Rhétorique française à l'usage des jeunes demoiselles, Bossange 1792, p. 261, http://gallica.bnf.fr/ark:/12148/bpt6k49071g (accès : 11.12.2020).

Faerber J., L'air de Rien, ou la suspension de la langue dans le Minimalisme en général et chez Christian Oster en particulier [dans :] Christian Oster et « Cie ».

${ }^{113}$ F. Wagner, La Vérité sur Jean-Philippe (Éléments pour une poétique de l'œuvre toussaintienne), «Vox-poetica. Lettres et sciences humaines » 2011, http://www.vox-poetica.org/t/articles/wagner2011. html (accès : 11.12.2020).

114 L. Demoulin, op. cit., p. 138-139.

115 M. Motkowicz, op. cit., p. 8-11 ; R. Amar, op. cit., p. 190, 192. 
Retour $d u$ romanesque, dir. A. Mura-Brunel, Amsterdam-New York 2006, p. 65-75.

Fontanier P., Les figures du discours, Paris 1977.

Gabriel J.-B., Fuir l'image avec désinvolture. Autour du court métrage Fuir de JeanPhilippe Toussaint, « Textyles : revue des lettres belges de la langue française » 2010, n $^{\circ} 38$, https://journals.openedition.org/textyles/289 (accès : 11.12.2020).

Genette G., Figures III, Paris 1972.

Glasco S., Parody and Palimpsest. Intertextuality, Language and the Ludic in the Novels of Jean-Philippe Toussaint, New York 2015.

Godard H., Le roman modes d'emploi, Paris 2006.

Hyppolite J.-L., Jean-Philippe Toussaint : Reticent Narratives [dans :] idem, Fuzzy Fiction, Nebraska 2006, p. 24-61.

Ingarden R., L'oeuvre d'art littéraire, Lausanne 1983.

Jankélévitch V., L'Ironie, Paris 1964 [1950].

Kaprèlian N., Rencontre avec Jean-Philippe Toussaint, https://www.lesinrocks. com/2009/09/25/actualite/actualite/rencontre-avec-jean-philippe-toussaint/ (accès : 11.12.2020).

Kibédi Varga A., Le récit postmoderne, «Littérature »1990, n 77, p. 3-22.

Korwin-Piotrowska D., Białe znaki. Milczenie w strukturze i znaczeniu utworów narracyjnych, Kraków 2015.

Le Petit Robert de la Langue française, 2011, informatisé, entrée « réticence », disque CD (accès : 11.12.2020).

Le site internet de Jean-Philippe Toussaint, http://www.jptoussaint.com/france.html (accès : 11.12.2020).

Le Trop et le trop peu, une esthétique des extrêmes, dir. P. Ouellet, S. Pépin, Montréal 1999.

Leclerc Y., Autour de Minuit, « Dalhousie French Studies »1989, Fall/Winter, p. 63-74.

Loignon S., Romanesque : le retour de flamme, ou comment faire l'amour avec J.-P. Toussaint? [dans :] Christian Oster et « Cie ». Retour du romanesque, dir. A. Mura-Brunel, Amsterdam-New York 2006, p. 25-34.

Meurée Ch., Le temps à l'épreuve du « désastre infinitésimal » [dans :] Les Vérités de Jean-Philippe Toussaint, dir. S. Chaudier, Saint-Étienne 2016, p. 201-210.

Meurée Ch., Thiry M., L'Autoportrait de l'artiste en éternel décalé, «Textyles : revue des lettres belges de la langue française » 2018, $\mathrm{n}^{\circ} 53$, https://journals.openedition. org/textyles/2931 (accès : 11.12.2020).

Motkowicz M., «La Salle de bain » de Jean-Philippe Toussaint en tant que roman minimaliste, mémoire de maîtrise non-publié, Cracovie 2011.

Motte W., Small Worlds. Minimalism in Contemporary French Literature, Nebraska 1999.

Mura-Brunel A., "Retour en grâce » de la fiction : "Un soir au club » de Christian Gailly et «Faire l'amour » de Jean-Philippe Toussaint [dans :] Romanciers minimalistes 1979-2003, dir. M. Dambre, B. Blanckeman, Paris 2012, p. 295-307.

Quintilien, Institutio oratoria, IX, III. 
Rapak W., Entre langage et silence - discours allusif [dans :] L'allusion en poésie, dir. J. Lajarrige, Ch. Moncelet, Clermont-Ferrand 2002, p. 49-61.

Richir A., Capter l'image : l'expérience du temps dans « L'Appareil-photo » et " Faire l'amour » [dans :] Les Vérités de Jean-Philippe Toussaint, dir. S. Chaudier, SaintÉtienne 2016, p. 177-188.

Richir A., Écriture du fantasme chez Jean-Philippe Toussaint et Tanguy Viel. Diffraction littéraire de l'identité, Louvain 2015.

Robbe-Grillet A., Pour un nouveau roman, Paris 1961.

Ruffel L., Le minimal et le maximal ou le renouvellement [dans :] Romanciers minimalistes 1979-2003, dir. M. Dambre, B. Blanckeman, Paris 2012, p. 43-54.

Ruffel L., Le temps des spectres [dans :] Le roman français aujourd'hui. Transformations, perceptions, mythologies, dir. B. Blanckeman, J.-Ch. Millois, Paris 2004, p. 95-115.

Shoots F., "Passer en douce à la douane ». L'Écriture minimaliste de Minuit : Deville, Echenoz, Redonnet et Toussaint, Amsterdam-Atlanta, GA 1997.

Toussaint J.-Ph., La Main et le Regard. LIVRE/LOUVRE, Paris 2012.

Toussaint J.-Ph., La Réticence, Paris 1991.

Toussaint J.-Ph., La Télévision, Paris 1997, p. 7-270.

Viart D., Vercier B., La littérature française au présent, Paris 2005.

Wagner F., D'un retour de flamme pour la fiction romanesque. La narration buissonnière selon Jean Echenoz, «Itinéraires » 2013, n 1, p. 29-49.

Wagner F., La Vérité sur Jean-Philippe (Éléments pour une poétique de l'œuvre toussaintienne), « Vox-poetica. Lettres et sciences humaines » 2011, http:/www.voxpoetica.org/t/articles/wagner2011.html (accès : 11.12.2020).

Wagner F., Retours, tours et détours du récit. Aspects de la transmission narrative dans quelques romans français contemporains, « Poétique » 2011, n 165, p. 3-20.

Zhao J., L'éloge de l'oisiveté [dans :] Les Vérités de Jean-Philippe Toussaint, dir. S. Chaudier, Saint-Étienne 2016, p. 93-100. 